\title{
Análise e interpretação de ideias: proposta de um modelo
}

Marina Carradore Sérgio ${ }^{I}$

http://orcid.org/0000-0002-3942-2249

http://dx.doi.org/10.1590/1981-5344/3306

As ideias são consideradas a matéria-prima do processo de inovação. Boas ideias podem impactar no planejamento de ações de melhoria contínua e na promoção de produtos diferenciados. O conhecimento envolvido no domínio de Gestão de Ideias é geralmente latente e deve ser descoberto de forma adequada usando métodos quantitativos, como a mineração de dados ou abordagens baseadas em dados. O grande desafio está em gerar ideias pragmáticas e úteis para $o$ desenvolvimento de novos produtos, faltando pesquisas para desenvolver completamente novos serviços ou ideias de produtos utilizando abordagens quantificadas ou orientadas a dados. Com o intuito de conceber um sistema para sustentação e facilitação deste processo é necessário formalizar os principais conhecimentos envolvidos no domínio. A representação e a formalização do conhecimento podem ocorrer semanticamente. $O$ objetivo deste estudo é propor um modelo de mineração de ideias, capaz de contribuir na análise e interpretação dos dados, com o intuito de auxiliar no processo de tomada de decisão. 
Palavras-chave: Gestão de Ideias; Mineração de ideias; Inovação; Modelo de mineração de ideias.

\title{
Analysis and interpretation of ideas: proposal of a model
}

\begin{abstract}
Ideas are considered the raw material of the innovation process. Good ideas can impact the planning of continuous improvement actions and the promotion of differentiated products. The knowledge involved in the domain of idea management is generally latent and must be adequately discovered using quantitative methods such as data mining or data-based approaches. The great challenge is to generate pragmatic and useful ideas for the development of new products, lacking research to completely develop new services or product ideas using quantified or data-oriented approaches. In order to design a system to support and facilitate this process it is necessary to formalize the main knowledge involved in the domain. The representation and formalization of knowledge can occur semantically. The objective of this study is to propose a model of idea mining, capable of contributing to the analysis and interpretation of the data, with the purpose of assisting in the decision making process.
\end{abstract}

Keywords: Idea management; Idea mining; Innovation; Idea mining model.

Recebido em 10.10.2017 Aceito em 22.03.2019

\section{Introdução}

Uma ideia consiste em algo que ainda não foi desenvolvido, comprovado e realizado (GURTEEN, 1998). Para Michalko (2003) uma ideia é caracterizada por um pensamento produtivo, é olhar as mesmas coisas sob uma nova linha de pensamento em busca de novas alternativas. Tidd, Bessant e Pavitt (2005) defendem que ideias são conceitos ou pensamentos transformados em artefato tangível. Para Koen et al. (2002), é a solução inicial para um problema elaborado. 
O processo de Gestão de Ideias é aplicado nas organizações a fim de promover inovações. Gerar ideias constitui desde o ato de identificar e analisar oportunidades externas e internas. Contudo, a Gestão de Ideias é - processo de captura, armazenamento e organização de ideias empregadas no processo avançado do front-end (MARTINI; NEIROTTI; APPIO, 2017).

Martini, Neirotti e Appio (2017) salientam que o processo de Gestão de Ideias permite que organizações confiem na criatividade, na atitude empresarial e na motivação de todos os funcionários e colaboradores. Mais especificamente, as práticas de Gestão de Ideias podem ser conceitualizadas como o processo de reconhecer a necessidade de ideias, subsidiar, gerar, avaliar e priorizar (FLYNN et al., 2003; VANDENBOSCH et al., 2006; GAMLIN; YOURD; PRATICK, 2007).

Atualmente, altas taxas de inovações falham. Isso, devido principalmente a seleção de ideias não inovadoras como ponto de partida (THORLEUCHTER; VAN DEN POEL, 2016). Nisto, o processo de inovação fica comprometido, gerando desperdício de tempo e custo (DISSELKAMP, 2015). Portanto, os tomadores de decisão na área da gestão da inovação necessitam de estimativas sobre o potencial inovador de uma ideia (THORLEUCHTER; VAN DEN POEL, 2016).

Este cenário apresenta grandes desafios em gerar ideias pragmáticas e úteis para a criação de novos produtos. Karimi-Majd e Mahootchi (2015) destacam que faltam pesquisas no âmbito de como criar novos serviços ou ideias de produtos usando abordagens quantificadas ou orientadas a dados. Hossain e Islam (2015a) afirmam que o grande desafio da área é avaliar as ideias e encontrar uma equipe apropriada para a avaliação das ideias, especialmente quando o volume de ideias é alto e necessita de avaliação em um curto período de tempo. Muitas organizações enfrentam dificuldades no gerenciamento do processo de avaliação e identificação de ideias recebidas de multidões (BOUDREAU; LAKHANI, 2013).

Objetivando desenvolver um sistema capaz de sustentar e facilitar este processo, é necessário formalizar os principais conhecimentos envolvidos no domínio. A representação e a formalização do conhecimento podem ser feitas através da semântica. Ontologias possibilitam estruturar as relações semânticas e podem ser definidas como um conjunto de termos ordenados hierarquicamente para representar um domínio específico, podendo ser utilizadas na representação da base de conhecimento onde são executados processos de inferência (raciocínio).

A ontologia é uma forma de representação caracterizada como "uma especificação formal e explícita de uma conceituação compartilhada" (STUDER; BENJAMINS; FENSEL, 1998). Segundo Gobin (2012) esta definição é baseada na ideia de conceitualização, uma visão simplificada do mundo real que se deseja representar, fornecendo uma conceitualização comum e compartilhada de um domínio, que pode ser comunicada entre pessoas e sistemas.

Ontologias são modelos conceituais utilizados para representar determinado domínio, interpretável por máquina, através de técnicas de 
representação de conhecimento (GRIMM; HITZLER; ABECKER, 2007). Segundo Chandrasekaran, Josephson e Benjamins (1998) ontologias ilustram a estrutura de conhecimento e permitem o compartilhamento. Por intermédio da ontologia é possível melhorar a gestão da informação (HERNÁNDEZ-GONZÁLEZ et al., 2014).

O objetivo deste estudo é propor um modelo de mineração de ideias, capaz de contribuir na análise e interpretação dos dados coletados em plataformas de Gestão de Ideias, com o intuito de auxiliar no processo de tomada de decisão.

O artigo está estruturado da seguinte forma: na Seção 1 é realizada uma introdução ao tema da pesquisa. Posteriormente, apresentam-se os conceitos relativos à Ideia, Gestão de Ideias, Mineração de Ideias, caracterizando a fundamentação teórica (Seção 2). Em seguida, Seção 3, apresenta-se o modelo proposto. Na Seção 4 são elucidadas algumas das técnicas e ferramentas utilizadas na mineração de ideias. Por fim, as considerações finais referentes ao trabalho.

\section{Mineração de ideias}

A competitividade presente no mercado exigiu dos tomadores de decisão, incluindo os investidores e suas equipes gerenciais, a necessidade de perceber exigências não ditas dos clientes (KARIMI-MAJD; MAHOOTCHI, 2015). Objetivando superar os desafios presentes no mercado, as organizações estão mudando o formato como conduzem o processo de inovação. Contudo, desenvolver a habilidade de produzir inovações e gerir as relações intra-organizacionais são fatores críticos para o sucesso do negócio (POORKAVOOS et al., 2016).

Ideias são à base do processo de inovação. Empresas como a Dell e a Lego, por exemplo, tem incentivado a criação de comunidades de inovação online. Através das plataformas de Gestão de Ideias, as pessoas produzem e criam ideias que podem contribuir no desenvolvimento de novos produtos, serviços, formação e promulgação de novas políticas (KHAN et al., 2014).

Contudo, as organizações ao desenvolverem esta abordagem podem ser confrontadas com o alto volume de ideias advindas da comunidade de usuários e enfrentar o problema de não conseguir filtrar e selecionar as ideias mais promissoras a serem desenvolvidas (POETZ; SCHREIER, 2012).

Para selecionar as ideias potencias perante o volume, os tomadores de decisão necessitam identificar as informações textuais-chave que representam as ideias e também considerar as opiniões dos colaboradores como comentários e votos sobre estas ideias (DINH et al., 2015). A identificação manual das ideias é considerado um processo dispendioso e demorado, devido o volume (DINH et al., 2015).

DINH et al. (2015) destacam a dificuldade do processo de avaliação e a relativa escassez de estudos de casos envolvendo tais plataformas, devido a produção de grandes conjuntos de dados não estruturados. 0 número de ideias produzidas na escala da multidão excede a capacidade 
das pessoas em processar informações, gastando-se muito tempo compreendendo o grande volume de ideias (CHAN; DANG; DOW, 2016).

Para descobrir padrões latentes em grandes volumes de dados, nos últimos anos emergiram as técnicas de mineração de dados, caracterizadas como ferramentas poderosas (KARIMI-MAJD; MAHOOTCHI, 2015). Este processo, também denominado de descoberta de conhecimento em bancos de dados (KDD) baseia-se na extração do conceito de palavras ocultas em textos ou documentos (ALKSHER et al., 2016).

O conceito de Mineração de Ideias foi introduzido por Thorleuchter (THORLEUCHTER, 2008; THORLEUCHTER; VAN DEN POEL; PRINZIE, 2010; DINH et al., 2015) e corresponde ao processo automático de extrair ideias novas e úteis de textos não estruturados (THORLEUCHTER, 2008; THORLEUCHTER; VAN DEN POEL; PRINZIE, 2010; DINH et al., 2015). O processo de extração de ideias a partir de textos depende dos padrões gerados, modelos, direções ou outras regras para textos não estruturados interessantes e úteis. Geralmente, o processo pode ser visto como uma extensão da mineração de texto por derivar informações de alta qualidade de texto não estruturado, o que também mostra a grande influência da extração de texto (ALKSHER et al., 2016).

O processo de mineração de ideias compõe-se de três etapas: preparação da descrição de problema, extração de padrões de texto de um novo texto e avaliação de padrões do texto relacionados à novidade e utilidade em relação à descrição do problema.

A mineração de ideias, segundo Alksher et al. (2016), é um campo novo e interessante na área de pesquisa de recuperação de informação. A literatura define como uma abordagem para extrair ideias potenciais de informações textuais utilizando ferramentas de mineração de texto (THORLEUCHTER; HERBERZ; VAN DEN POEL, 2011).

Os autores Alksher et al. (2016) ressaltam que as ideias expostas no formato textual, podem ser expostas em diferentes formatos como publicações em blogs e até mesmo como patentes. As ideias são úteis para o público e para o tomador de decisões, sendo crescente e recente o interesse de pesquisadores pela área de mineração de ideias (ALKSHER et al., 2016).

Ao aplicar a mineração de ideias se extrai e se descobre fontes de informações não estruturadas que podem levar a identificação de ideias potenciais e contribuir com os tomadores de decisões estratégicas (ALKSHER et al., 2016). Os autores ressaltam que no processo de avaliação, a literatura científica sugere que a intervenção humana é necessária para entender o conceito de composição do texto. Embora a extração de ideias por um especialista humano seja muito demorada e onerosa, o especialista também identifica ideias novas e úteis manualmente com alta precisão sem usar a abordagem de mineração de ideias.

Segundo Thorleuchter e Van Den Poel (2012) a pesquisa na área de mineração de ideias mostra que a qualidade dos resultados depende 
fortemente do domínio. Isso ocorre porque ideias de diferentes domínios consistem em diferentes propriedades.

\section{Modelos de mineração de ideias}

Ao realizar uma revisão da literatura nas bases de dados Scopus, Science Direct e IEEE Xplore foram identificados 19 artigos contendo os termos de busca ("idea mining" and "model"). Destes, apenas 6 correspondem ao tema desta pesquisa.

No artigo intitulado " $A$ review of methods for mining idea from text", os autores Alksher et al. (2016), propuseram um modelo, apresentado na Figura 1, composto por 5 etapas.

Figura 1- Modelo proposto por Alksher et al. (2016)

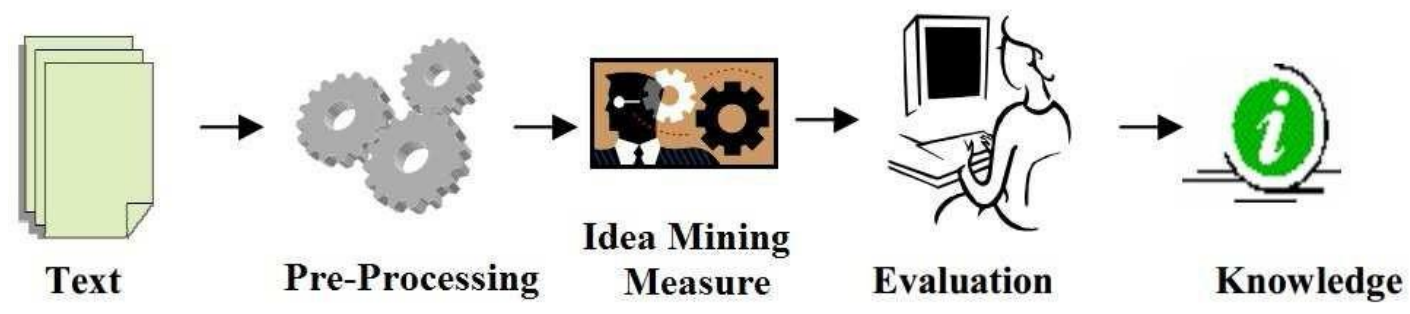

Fonte: (ALKSHER et al., 2016).

Alksher et al. (2016) identificaram diferentes modelos de processamento de ideias com formas para caracterizar, extrair, criar e avaliar ou então abordagens voltadas a criação, no qual considera-se o problema e cria-se novas ideias relacionadas ao problema, utilizando métodos de mineração de texto, e finalizando com o processo de avaliação das ideias criadas.

No modelo proposto pelos autores, o processo consiste em três etapas necessárias, sendo: 1) Preparação do documento coletado: foca-se no texto do problema; 2) Extração de padrões de texto (ideias) a partir de um novo documento de texto. Os padrões de texto são comparados com a descrição do problema utilizando uma medida de mineração de ideias. E, por último a avaliação dos padrões de texto, avaliando-os quanto à sua usabilidade e utilidade.

No modelo proposto por Mehtälä et al. (2016), os autores apresentam um novo modelo de processos de crowdsourcing que é especialmente adequado para a indústria da moda. A Figura 2 apresenta o modelo. 


\section{Finira 2 Mndoln nrnnncto nnr Mohtälä ot al. (2016)}
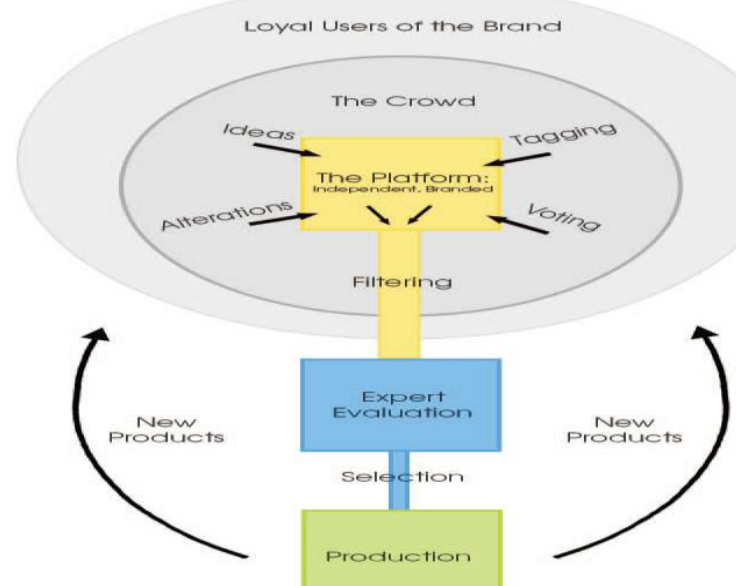

Fonte: (MEHTÄLÄ et al., 2016).

Os autores destacam que a pré-avaliação executada pela multidão é crucial neste campo para o gerenciamento eficiente de todos os projetos que a plataforma produz, motivando os usuários das plataformas através de um sistema de classificação e pontos. A votação da multidão é utilizada para filtrar as ideias com maior demanda, passando por uma avaliação de especialistas.

Thorleuchter, Van Den Poel e Prinzie (2010) apresentam um modelo para mineração de ideias como sendo um processo de extração de ideias novas e úteis a partir de textos não estruturados. A abordagem de mineração de ideias proposta, extrai automaticamente ideias de textos fornecidos pelo usuário. A Figura 3 apresenta o modelo.

Figura 3- Metodologia proposta por Thorleuchter, Van Den Poel e Prinzie (2010) 
New Text

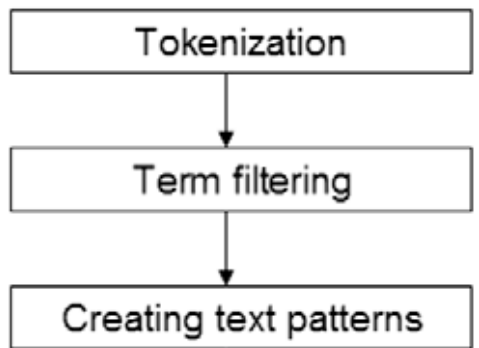

Creating term vectors
Problem description

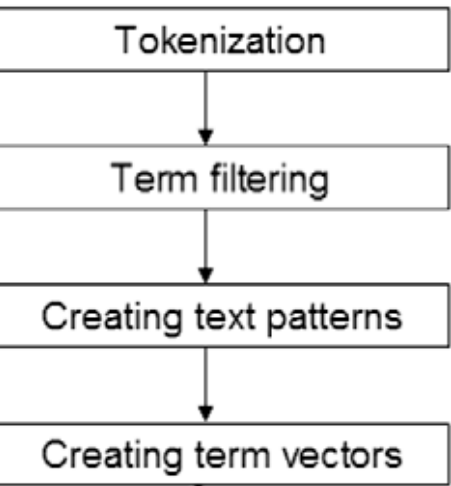

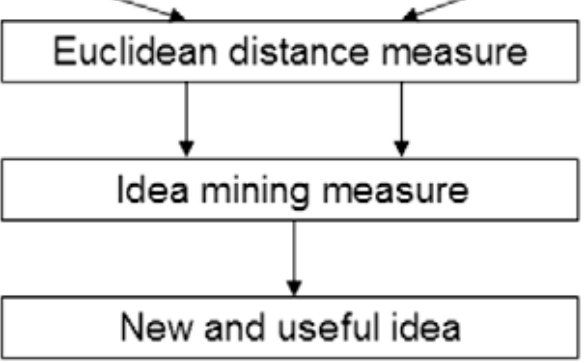

Fonte: (THORLEUCHTER; VAN DEN POEL. PRINZIE, 2010).

O processamento da abordagem de mineração de ideias ocorre em diferentes etapas: após a tokenização e a filtragem de termos, os padrões de texto são criados e os vetores de termos são construídos para representar os padrões. Os vetores temporários do novo texto são comparados aos vetores de termos a partir da descrição do problema, usando a medida de distância euclidiana. Em seguida, os vetores de termos do novo texto são comparados aos vetores de termos mais semelhantes da descrição do problema usando uma medida de mineração de ideias. Como resultado, obtêm-se vetores de termos do novo texto que representa ideias novas e úteis.

Thorleuchter e Van Den Poel (2013) apresentam uma metodologia, conforme a Figura 4.

Figura 4- Metodologia proposta por Thorleuchter e Van Den Poel (2013) 


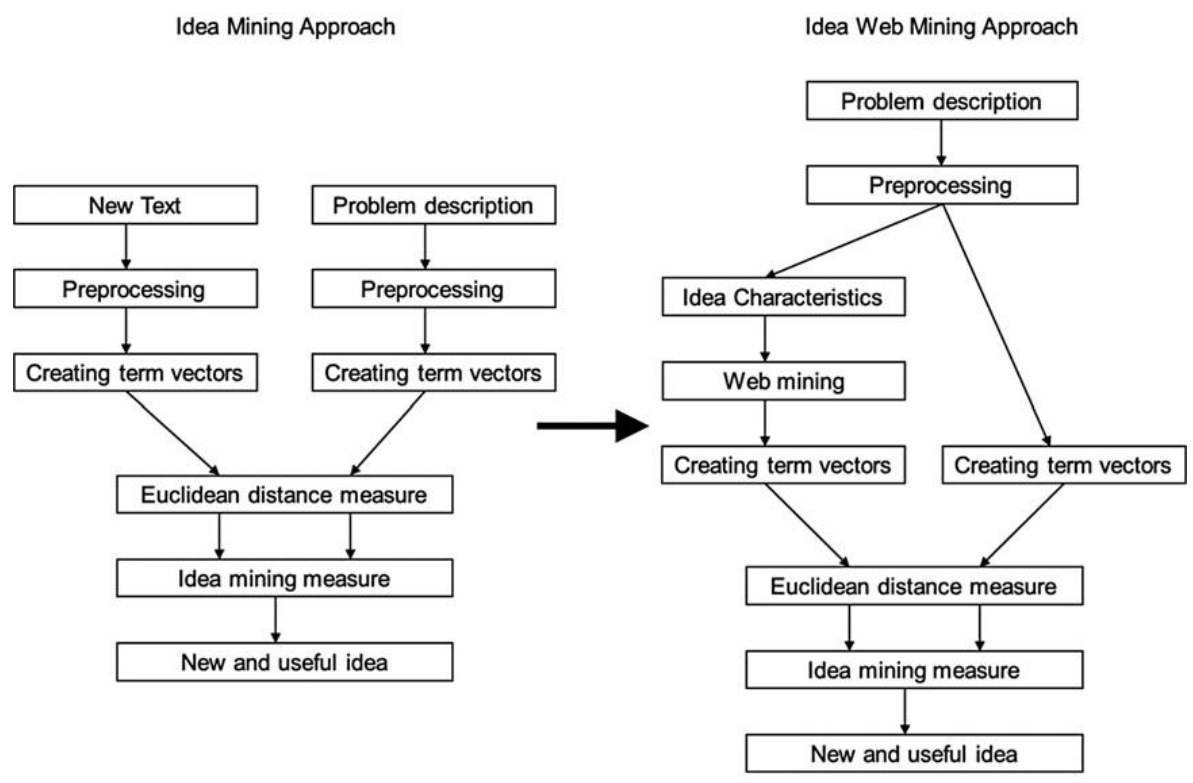

Fonte: (THORLEUCHTER; VAN DEN POEL, 2013).

O processamento da ideia ocorre com base na abordagem de mineração web (lado direito da Figura 4) e com base no processamento na abordagem de mineração de ideias (lado esquerdo da Figura 4). O usuário fornece um contexto (descrição do problema). Após o préprocessamento, os vetores de termos são gerados e as consultas de pesquisa são criadas representando o problema. As consultas são executadas por um mecanismo de pesquisa na web e os vetores de termos são criados a partir dos resultados da consulta com base nas características específicas da ideia de domínio. Eles são comparados aos vetores de termos do contexto usando a medida de distância euclidiana e a medida de mineração de ideias. Como resultado, ideias novas e úteis de solução de problemas são extraídas da internet.

Thorleuchter e Van Den Poel (2015) propõem uma metodologia para mineração de ideias, com a proposição de um algoritmo que identifica padrões textuais relevantes de documentos ou sites para resolver um determinado problema estratégico. Segundo os autores, para aplicar a mineração de ideias um usuário deve fornecer um texto com a descrição do problema. O usuário também tem que fornecer outros dados textuais. A mineração de ideias identifica um padrão textual a partir do texto da ideia como uma nova ideia (que pode ser usada para resolver um problema existente).

A abordagem proposta está representada na Figura 5, tendo como objetivo identificar novos sinais emergentes no ambiente organizacional com relevância para um determinado problema de decisão estratégica da organização. Assim, uma descrição do problema de decisão deve ser fornecida em forma de texto simples. As palavras-chave devem ser incluídas no texto e o problema deve ser abordado de forma clara e compreensível. Esses dados de entrada são processados em várias etapas. Um vetor de pré-processamento de termos é criado. Transformam-se os dados recebidos do passo anterior para um formato 
específico conforme exigido pela etapa subsequente. Os métodos padrões da mineração de texto são usados nesta etapa. A mineração web é aplicada para identificar se a informação textual a partir da Internet está relacionada com o determinado problema estratégico. Criam-se e executam-se consultas de pesquisa com base nas palavras-chave fornecidas e rastreia-se o texto completo da recuperação. Como resultado, é dada uma coleção de documentos da internet representando o ambiente da organização. A etapa de mineração de ideias compara a descrição do problema com a coleção de documentos resultantes da etapa de mineração web. Com isto, identificam-se novas ideias dentro da coleção que podem ser capazes de resolver o determinado problema de decisão estratégica. Com base nas ideias identificadas analisasse o sinal fraco. Neste momento, agrupam-se as novas ideias, identificam-se os sinais fracos, e se fornece sinais fracos ao especialista para melhorar a tomada de decisão estratégica. O desempenho geral da metodologia proposta é calculado na etapa de avaliação. Medidas de padrão de desempenho são utilizadas para mostrar a precisão e a recuperação dos resultados com base em uma avaliação manual por especialistas humanos.

Figura 5- Metodologia proposta por Thorleuchter e Van Den Poel (2015)

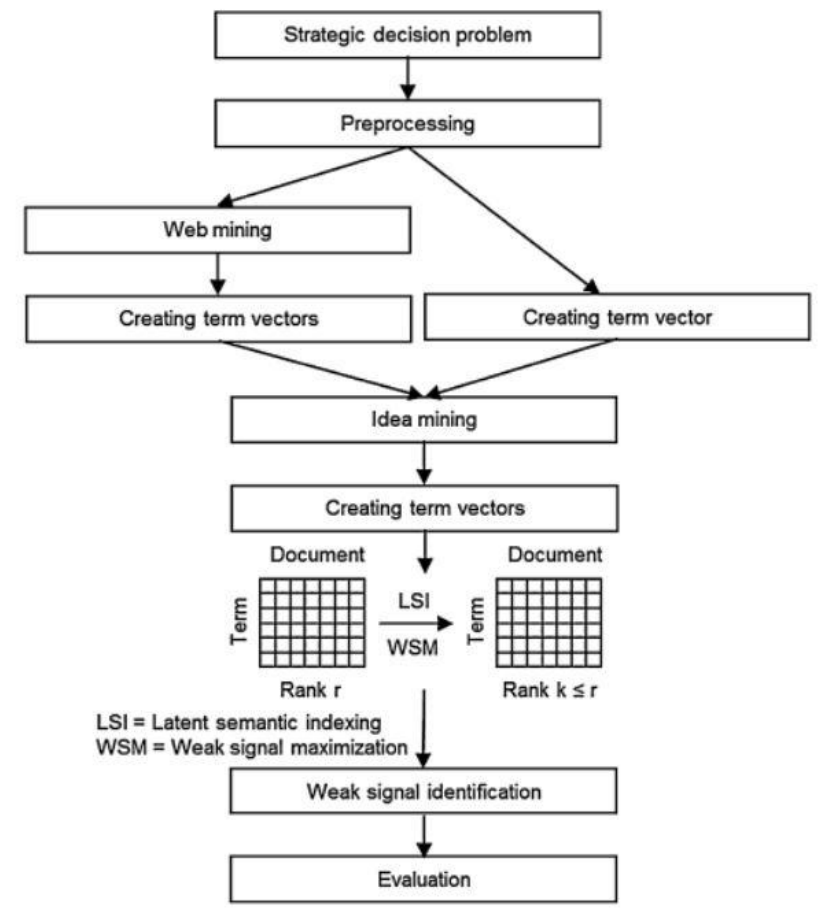

Fonte: (THORLEUCHTER; VAN DEN POEL, 2015).

Liu, Goulding e Brailsford (2015) propõem uma metodologia representada na Figura 6 , centrada na descoberta de relações entre problema/solução. A análise ocorre sobre frases detectadas em resumos de estudos científicos. O resumo é considerado como uma descrição total e autossuficiente. As noun-phrases que contém o resumo devem refletir a(s) questão(ões) que o(s) autor(es) desejam abordar. Nisto, uma lista de noun-phrases é extraída do resumo e serve como um pool de sementes 
de $p$-phrases. Se uma s-phrase é detectada no mesmo texto, as relações semânticas entre ela e as $p$-phrases são estabelecidas.

Com base nesta premissa, esta abordagem pode ser dividida em seis etapas: 1) Extração de Noun-phrase de um corpo corporativo de treinamento usando a marcação; 2) Filtragem de phrase para remover stopwords e texto com baixo conteúdo de informações; 3) Classificação das noun-phrases em p-phrases (problemas) e s-phrases (soluções); 4) Agregação de triplas de ideias altamente co-ocorrentes <p-phrase, $s-$ phrase, relevance $>$; 5) Processamento deste conjunto de triplas de ideias conhecidas através de um mecanismo de filtragem colaborativa; 6) Avaliação da lista classificada de ideias inovadoras.

Figura 6- Metodologia proposta por Liu, Goulding e Brailsford (2015)

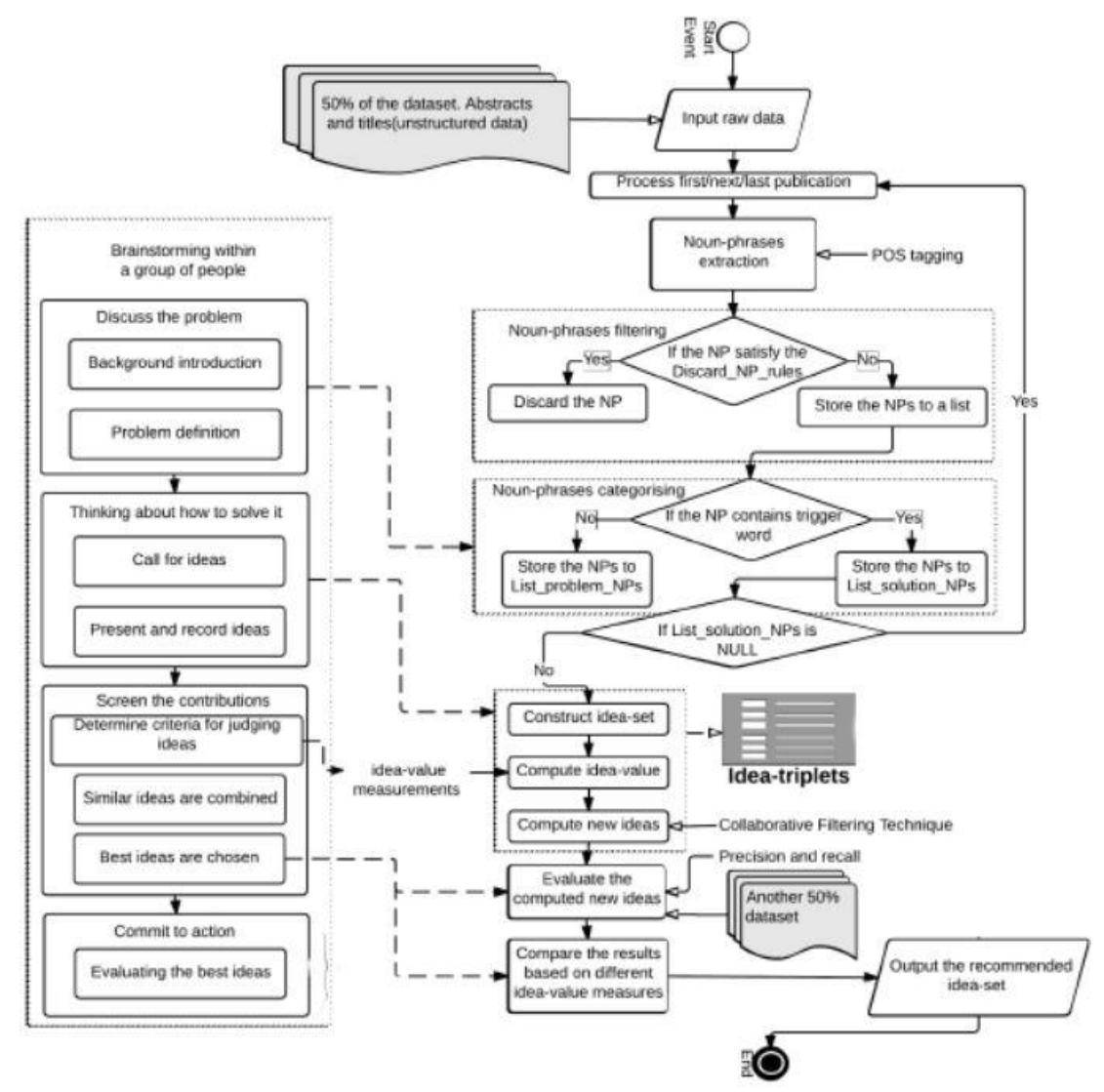

Fonte: (LIU; GOULDING; BRAILSFORD, 2015).

\section{Modelo proposto}

Os modelos de mineração de ideias podem variar nos seus detalhes. O modelo proposto consiste em 5 etapas fundamentais: a seleção, o préprocessamento, a transformação, a aplicação das técnicas de mineração e a análise e interpretação das ideias. A Figura 7 apresenta 0 modelo proposto. 
Figura 7 - Modelo de mineração de ideias

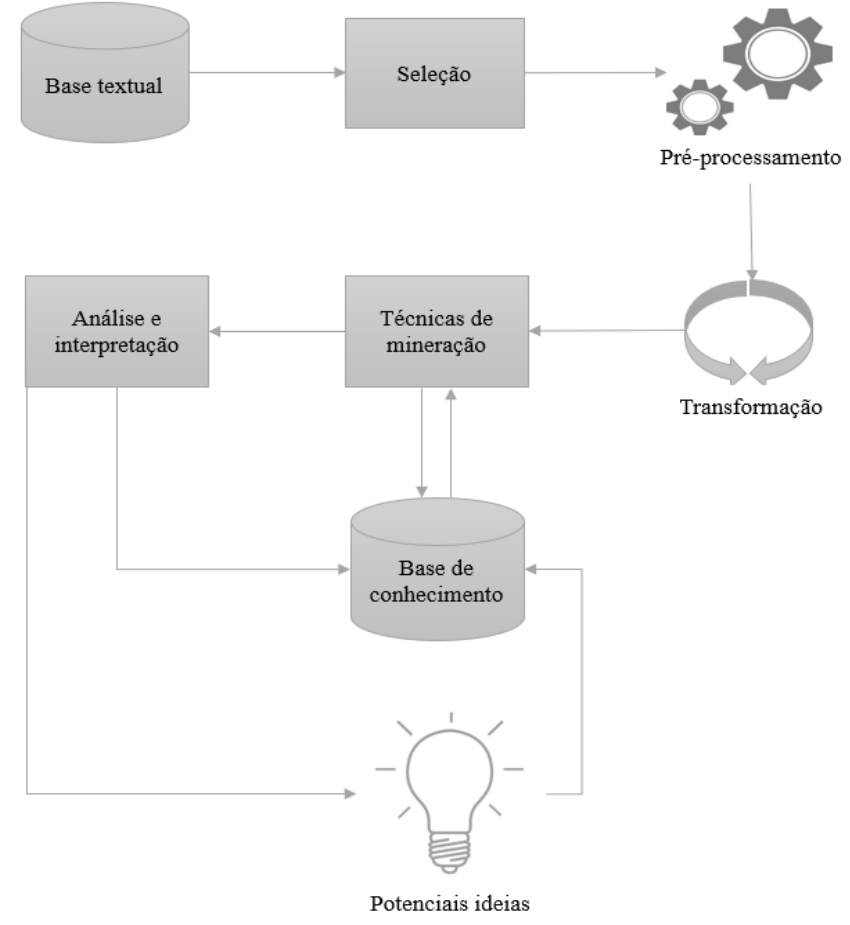

Fonte: Autores.

O modelo inicia com a formação da base textual, caracterizada por documentos textuais. Posteriormente, é realizada a etapa de seleção com base em filtros estabelecidos por especialistas e estabelece-se a descrição do problema que será analisado.

$\mathrm{Na}$ etapa seguinte, é realizado o pré-processamento tendo como objetivo prover a formatação e representação do formato textual das ideias. É bastante onerosa, com a aplicação de diversos algoritmos que consomem boa parte do tempo do processo de extração de conhecimento. Nesta etapa, também ocorrem as consultas em fontes externas com o intuito de auxiliar no processo de decisão. Consultas estas que pode ser realizadas na web, em bases de patentes, em relatórios expedidos por organizações com as tendências na área de negócios e investimentos. O objetivo destas consultas é criar novas ideias relacionadas ao problema, além de identificar potenciais áreas de investimento, produtos similares que já foram desenvolvidos, entre outros. A análise em bases de patentes pode gerar indicadores de originalidade, por exemplo. Estas informações serão agregadas ao processo de mineração e possivelmente contribuíram no processo de avaliação de interpretação do domínio.

Posteriormente, ocorre a etapa de transformação. Esta etapa consiste na adequação dos dados para serem utilizados em algoritmos de extração de padrões. Estas transformações podem variar conforme o domínio da aplicação.

Em seguida, aplicam-se técnicas de mineração objetivando descobrir padrões úteis que possam contribuir no processo de avaliação. Os vetores de termos identificados na etapa de pré-processamento são 
comparados a descrição do problema utilizando uma medida de mineração de ideias. Esta etapa é responsável pelo desenvolvimento de cálculos, inferências e algoritmos, tendo como intuito a extração de conhecimento e a descoberta de padrões. Como entrada para o processo de mineração realiza-se também uma busca na base de conhecimento com o intuito de identificar possíveis características que a organização considera importantes para tomar a decisão na escolha das ideias a serem selecionadas. Esta base de conhecimento pode ser representada no formato de uma ontologia. Dentre as características presentes na base podem estar restrições e também caraterísticas relacionadas ao contexto organizacional, fontes de financiamento, critérios de seleção como viabilidade, originalidade, aplicabilidade. As características e os padrões identificados são então comparados às ideias.

Finalmente, realiza-se a análise e interpretação das informações por especialistas da organização e elucidam-se as potenciais ideias descobertas quanto a sua utilidade e aplicabilidade. O conhecimento descoberto na etapa de mineração é armazenado na base de conhecimento da organização bem como as ideias potenciais. A base de conhecimento armazenará o resultado da análise de polaridade, que consiste em determinar a polaridade da opinião do especialista em relação à ideia, verificando, por exemplo, se a ideia é favorável à implementação, neutra ou negativa, em relação a esse quesito.

O modelo proposto nesta pesquisa comparado aos apresentados na seção anterior possibilita retroalimentar a base de conhecimento. A base de conhecimento tem como intuito armazenar possíveis características fundamentais a organização e ao processo de tomada de decisão, colaborando na escolha das ideias a serem selecionadas. Como mencionado anteriormente, a base de conhecimento pode ser modelada no formato de uma ontologia. Esta ontologia é responsável por representar o conhecimento de domínio, axiomas e características que contribuem na tomada de decisão. Dentre estas características podem estar restrições e também caraterísticas relacionadas ao contexto organizacional, fontes de financiamento, critérios de seleção como viabilidade, originalidade, aplicabilidade e/ou áreas estratégicas que a organização gostaria de investir. As características e os padrões identificados são então comparados às ideias identificadas a partir dos textos. O modelo proposto torna-se mais robusto em termos de consultas e pesquisas e permite a realização de inferências sobre os resultados obtidos com o processo de mineração, além de contribuir na construção de indicadores.

\section{Algumas técnicas e ferramentas empregadas na mineração de ideias}

Ao realizar um mapeamento da literatura sobre as ferramentas envolvendo a área de mineração de ideias foram identificados alguns trabalhos que serão citados a seguir no Quadro 1. 
Quadro 1 - Tecnologias e ferramentas utilizadas no domínio de Gestão de Ideias

\begin{tabular}{|c|c|}
\hline Ferramenta & Autor(es) \\
\hline Cluster & $\begin{array}{l}\text { Chan, Dang e Down } \\
\text { Niederberger et al. (2016); }\end{array}$ \\
\hline $\begin{array}{l}\text { Método semi-automático de mineração de } \\
\text { texto }\end{array}$ & Dinh et al. (2015) \\
\hline $\begin{array}{l}\text { Sistema de suporte à geração de ideias para } \\
\text { pessoas com conhecimentos escassos }\end{array}$ & Itou, Higashi e Munemori(2015) \\
\hline Método de avaliação de ideias & Murukannaiah, Ajmeri e Singh (2016) \\
\hline Mecanismo Note & Paukkeri e Kotro (2009) \\
\hline Plataforma ideaHound & Siangliulue et al. (2016) \\
\hline Plataforma de mineração & $\begin{array}{l}\text { Thorleuchter, Van Den Poel e Prinzie } \\
\text { (2010) }\end{array}$ \\
\hline Ferramenta de extração & Thorleuchter e Van Den Poel (2015) \\
\hline Metodologia de identificação de ideias & Thorleuchter e Van Den Poel (2016) \\
\hline Mapas de deliberação & Klein e landoli (2008) \\
\hline Tecnologias semânticas & Westerski, Iglesias e Rico (2010) \\
\hline $\begin{array}{l}\text { Métodos de processamento de linguagem } \\
\text { natural }\end{array}$ & Convertino, Sándor e Baez (2013) \\
\hline $\begin{array}{l}\text { Ferramenta para aplicação de mercados de } \\
\text { agregação de informações }\end{array}$ & Bothos, Apostolou e Mentzas (2012) \\
\hline Técnicas de análise de redes sociais & Björk e Magnusson (2009) \\
\hline
\end{tabular}

Fonte: Autores

A literatura apresenta diferentes abordagens envolvendo o domínio, mas sempre direcionadas a descoberta de padrões envolvendo informações não estruturadas.

\section{Considerações finais}

Os sistemas de Gestão de Ideias possibilitaram as organizações realizarem a captura de ideias fornecidas por diferentes atores. Um processo estruturado de seleção, avaliação e implementação de ideias produz maior agilidade e rapidez para levar novos produtos ao mercado. Estas plataformas são fundamentais para apoiar, estimular e facilitar o processo de Gestão de Ideias.

Este estudo apresentou uma visão geral sobre a mineração de ideias, que ganhou crescente atenção nos últimos anos. A expansão contínua de dados textuais levou à necessidade de metodologias de mineração e técnicas para analisar dados ocultos em textos não estruturados. A mineração de ideias é uma área de pesquisa aberta com ambiguidades na caracterização da ideia. Consequentemente, vários estudos adotaram técnicas de mineração de texto e aplicaram uma abordagem automática de mineração de ideias para extrair ideias que possam ser potenciais. Em geral, portanto, parece que a melhoria da criação de padrões textuais efetivamente possibilita a extração de ideias com uma combinação de medidas através da mineração de ideias. 
O modelo proposto possibilita inserir documentos textuais e realizar a análise com base em: consultas na web, bases de patentes, relatórios de tendências e investimentos, gerando insumos para indicadores; bem como em regras já concebidas pela organização; análise do contexto organizacional; áreas de investimentos desejadas; enfim, e assim proporcionar a visualização das ideias com maior potencial de investimento. O modelo torna-se mais robusto em comparação aos apresentados na literatura, pois engloba mais insumos, contribuindo no processo de tomada de decisão.

Outra característica importante do modelo é a presença da base de conhecimento, podendo ser representada no formato de uma ontologia. A ontologia é responsável por representar o conhecimento de domínio, axiomas e características que contribuem na tomada de decisão, bem como indicadores. Dentre estas características podem estar restrições e também características relacionadas ao contexto organizacional, fontes de financiamento, critérios de seleção como viabilidade, originalidade, aplicabilidade e/ou áreas estratégicas que a organização gostaria de investir. A ontologia é responsável por armazenar o resultado das consultas realizadas em base de patentes, na web, em relatórios de tendências e outros documentos. Tendo ainda a capacidade de gerar inferências sobre os resultados obtidos com o processo de mineração. Cabe ressaltar que a intervenção do especialista é fundamental para a avaliação do resultado do modelo. O especialista determinará se determinada ideia irá ou não ser implementada, e esta informação será armazenada na base de conhecimento, o que contribuirá na análise do desempenho do sistema.

Conceber e desenvolver plataformas voltadas à Gestão de Ideias torna-se uma tarefa complexa. Contudo, apesar dos potenciais benefícios do crowdsourcing, são necessárias maiores pesquisas sobre o suporte à tomada de decisões gerenciais.

\section{Referências}

ALKSHER, Mostafa A. et al. A review of methods for mining idea from text. In: INFORMATION RETRIEVAL AND KNOWLEDGE MANAGEMENT (CAMP), 2016 THIRD INTERNATIONAL CONFERENCE ON. IEEE, 2016. p. 88-93.

BJÖRK, Jennie; MAGNUSSON, Mats. Where do good innovation ideas come from? Exploring the influence of network connectivity on innovation idea quality. Journal of Product Innovation Management, v. 26, n. 6, p. 662670, 2009.

BOTHOS, Efthimios; APOSTOLOU, Dimitris; MENTZAS, Gregoris. A collaborative information aggregation system for idea management. In: INTERNET AND WEB APPLICATIONS AND SERVICES, 2008. ICIW'08. THIRD INTERNATIONAL CONFERENCE ON. IEEE, 2008. p. 289-296. 
BOUDREAU, Kevin J.; LAKHANI, Karim R. Using the crowd as an innovation partner. Harvard business review, v. 91, n. 4, p. 60-9, 140, 2013.

CHAN, Joel; DANG, Steven; DOW, Steven P. Comparing Different Sensemaking Approaches for Large-Scale Ideation. In: CHI CONFERENCE ON HUMAN FACTORS IN COMPUTING SYSTEMS, 2016. ACM

[Proceedings...].p. 2717-2728.

CHANDRASEKARAN, B., JOSEPHSON, J. R., BENJAMINS, R. Ontology of Tasks and Methods. In: WORKSHOP ON KNOWLEDGE ACQUISITION, MODELING AND MANAGEMENT (KAW'98), Banff, Canada. 1998.

CONVERTINO, Gregorio; SÁNDOR, A.; BAEZ, Marcos. Idea spotter and comment interpreter: Sensemaking tools for idea management systems. In: ACM COMMUNITIES AND TECHNOLOGIES WORKSHOP: LARGE-SCALE IDEA MANAGEMENT AND DELIBERATION SYSTEMS WORKSHOP. 2013.

DINH, Thanh-Cong et al. A framework to discover potential ideas of new product development from crowdsourcing application. INTERNATIONAL CONFERENCE ON COMPUTER, NETWORKS, SYSTEMS, AND INDUSTRIAL APPLICATIONS (CNSI 2012). 2015.

DISSELKAMP, Marcus. Innovations management: Instrumente und Methoden zur Umsetzung im Unternehmen. Zurich: Springer-Verlag, 2015.

FLYNN, Michael et al. Idea management for organisational innovation. International Journal of innovation management, v. 7, n. 04, p. 417-442, 2003.

GAMLIN, Janet N.; YOURD, Raymond; PATRICK, Valerie. Unlock creativity with "active" idea management. Research-Technology Management, v. 50, n. 1, p. 13-16, 2007.

GOBIN, B. A. Reusing OWL-S to model knowledge intensive tasks performed by Knowledge Based Systems. In: INTERNACIONAL CONFERENCE ON ICT AND KNOWLEDGE ENGINEERING, 2012, Bangkok, Tailândia. [Procedings...] IEEE, 2012. p. 34-42

GRIMM, S., HITZLER, P., ABECKER, A. Knowledge Representation and Ontologies: Logic, Ontologies and Semantic Web Languages. In: STUDER, Rudi; GRIMM, Grimm, ABECKER, Andreas(Eds). Semantic Web Services: Concepts, Technologies, and Applications. Zurich: Springer, 2007. p.3787.

GURTEEN, D. Knowledge, creativity and innovation. Journal of Knowledge Management, v.2, n.1, p.5-13, 1998

HERNÁNDEZ-GONZÁLEZ, Yolanda et al. A semantic-based platform for R\&D project funding management. Computers in Industry, v. 65, n. 5, p. 850-861, 2014. 
HOSSAIN, Mokter; ISLAM, KM Zahidul. Generating ideas on online platforms: A case study of "My Starbucks Idea". Arab Economic and Business Journal, v. 10, n. 2, p. 102-111, 2015 (a).

ITOU, Junko; HIGASHI, Takayuki; MUNEMORI, Jun. Proposal and Comparison of an Idea Generation Support System Presenting Words for the Use of Scarce Knowledge People. Procedia Computer Science, v. 60, p. 918-925, 2015.

KARIMI-MAJD, Amir-Mohsen; MAHOOTCHI, Masoud. A new data mining methodology for generating new service ideas. Information Systems and e-Business Management, v. 13, n. 3, p. 421-443, 2015.

KHAN, Khairullah et al. Mining opinion components from unstructured reviews: A review. Journal of King Saud University-Computer and Information Sciences, v. 26, n. 3, p. 258-275, 2014.

KLEIN, M.; IANDOLI, L. Supporting Collaborative deliberation using a large-scale argumentation: The MIT Collaboratorium. In: SYMPOSIUM ON DIRECTIONS AND IMPLICATIONS OF ADVANCED COMPUTING,11., 2008. [Proceedings...].2008.

KOEN, P. A. ; et al. Fuzzy front end effective methods, tools, and techniques. In: BELLIVEAU; A. P.; GRIFFIN; S. SOMERMEYER (Eds); The PDMA toolbook for new product development. New York: John Wiley, 2002. p.5-35.

LIU, Haixia; GOULDING, James; BRAILSFORD, Tim. Towards computation of novel ideas from corpora of scientific text. In: JOINT EUROPEAN CONFERENCE ON MACHINE LEARNING AND KNOWLEDGE DISCOVERY IN DATABASES. Springer. Cham, 2015. p.541-556.

MARTINI, Antonella; NEIROTTI, Paolo; APPIO, Francesco Paolo. Knowledge searching, integrating and performing: always a tuned trio for innovation? Long Range Planning, v. 50, n. 2, p. 200-220, 2017.

MEHTÄLÄ, Joanna et al. A crowdsourcing model for new idea development in the fashion industry. In: SERVICE OPERATIONS AND LOGISTICS, AND INFORMATICS (SOLI), 2016 IEEE INTERNATIONAL CONFERENCE ON. IEEE, 2016. p. 29-36.

MICHALKO, M. From bright ideas to right ideas: capturing the creative spark. The Futurist, v. 37, n. 5, p. 52-56, 2003.

MURUKANNAIAH, Pradeep K.; AJMERI, Nirav; SINGH, Munindar P. Acquiring creative requirements from the crowd: Understanding the influences of personality and creative potential in Crowd RE. In: REQUIREMENTS ENGINEERING CONFERENCE (RE), 24. 2016. IEEE, 2016. p. $176-185$.

NIEDERBERGER, Thomas et al. Hebbian principal component clustering for information retrieval on a crowdsourcing platform. In: NONLINEAR

DYNAMICS OF ELECTRONIC SYSTEMS, 2012. [Proceedings...]. VDE, 2012. p. 1-4. 
PAUKKERI, Mari-Sanna; KOTRO, Tanja. Framework for analyzing and clustering short message database of ideas. na, 2009.

POETZ, Marion K. ; SCHREIER, Martin. The value of crowdsourcing: can users really compete with professionals in generating new product ideas? Journal of Product Innovation Management, v. 29, n. 2, p. 245-256, 2012. POORKAVOOS, Meysam et al. Identifying the configurational paths to innovation in SMEs: A fuzzy-set qualitative comparative analysis. Journal of Business Research, v. 69, n. 12, p. 5843-5854, 2016.

SALDIVAR, Jorge et al. Idea Management Communities in the Wild: An Exploratory Study of 166 Online Communities. In: COLLABORATION TECHNOLOGIES AND SYSTEMS (CTS), 2016 INTERNATIONAL CONFERENCE ON. IEEE, 2016. p. 81-89.

SIANGLIULUE, Pao et al. IdeaHound: Improving Large-scale Collaborative Ideation with Crowd-powered Real-time Semantic Modeling. In: ANNUAL SYMPOSIUM ON USER INTERFACE SOFTWARE AND TECHNOLOGY, 29. 2016. [Proceedings...]ACM, 2016. p. 609-624.

STUDER, R.; BENJAMINS, V. R.; FENSEL, D. Knowledge engineering: principles and methods. Data \& knowledge engineering, v. 25, n. 1, p. 161-197, 1998.

THORLEUCHTER, Dirk. Finding new technological ideas and inventions with text mining and technique philosophy. In: DATA ANALYSIS, MACHINE LEARNING AND APPLICATIONS. Springer Berlin Heidelberg, 2008. p. 413420.

THORLEUCHTER, Dirk; HERBERZ, Sarah; VAN DEN POEL, Dirk. Mining social behavior ideas of Przewalski horses. In: ADVANCES IN COMPUTER, COMMUNICATION, CONTROL AND AUTOMATION. Springer, Berlin, Heidelberg, 2011. p. 649-656.

THORLEUCHTER, Dirk; VAN DEN POEL, Dirk. Extraction of ideas from microsystems technology. In: ADVANCES IN COMPUTER SCIENCE AND INFORMATION ENGINEERING, 2012. p. 563-568.

THORLEUCHTER, Dirk; VAN DEN POEL, Dirk. Idea mining for web-based weak signal detection. Futures, v. 66, p. 25-34, 2015.

THORLEUCHTER, Dirk; VAN DEN POEL, Dirk. Identification of interdisciplinary ideas. Information Processing \& Management, v. 52, n. 6, p. 1074-1085, 2016.

THORLEUCHTER, Dirk; VAN DEN POEL, Dirk. Web mining based extraction of problem solution ideas. Expert Systems with Applications, v. 40, n. 10, p. 3961-3969, 2013.

THORLEUCHTER, Dirk; VAN DEN POEL, Dirk; PRINZIE, Anita. Mining ideas from textual information. Expert Systems with Applications, v. 37, n. 10, p. 7182-7188, 2010. 
TIDD, J.; BESSANT, J.; PAVITT, K. Gestão da inovação. 3. ed. Porto Alegre: Bookman, 2005.

VANDENBOSCH, B.; SAATCIOGLU, A.; FAY, S. Idea management: a systemic view. Journal of Management Studies, Malden, v. 43, n. 2, p. 259-288, 2006.

WESTERSKI, Adam; IGLESIAS, Carlos Angel; RICO, Fernando Tapia. A Model for Integration and Interlinking of Idea Management Systems. In: MTSR. 2010. p. 183-194. 